\section{Gene-function wiki would let biologists pool worldwide resources}

SIR - Your Special Report ${ }^{\alpha}$ Internet encyclopaedias go head to head" (Nature $438,900-901 ; 2005$ ) shows that Wikipedia comes close to Britannica in terms of the accuracy of its science entries.

As a frequent user of Wikipedia and also a biologist, I hope that one day a wiki on gene function will be voluntarily created and maintained by biologists.

After a microarray experiment or a BLAST search, hundreds or thousands of interesting gene names are revealed, but the average biologist has no clue as to the function of most of them. Following up with a literature search wastes a lot of researchers' time and energy.

A wiki on gene function would make life very much easier for biologists. Such a wiki would also be less susceptible to spam, as most users would be biologists. It would also be more accurate, as long as statements are accompanied by references.

Several gene-function databases already exist, but each has certain disadvantages for the average biologist.

The Entrez Gene database (www.ncbi. nlm.nih.gov/entrez/query fcgi? $\mathrm{db}=$ gene) contains a Gene Reference Into Function, or GeneRIF, that allows users to submit statements with supporting literature references to annotate gene function. But although many short statements have accumulated in this database, it would be useful to have concise summaries compiled by experts, and these are not usually available.

The Molecule Pages for signalling proteins (www.signaling-gateway.org/molecule) and the OMIM database for human inherited diseases (www.ncbinlm.nih.gov/entrez/ query.fcgi? $\mathrm{db}=\mathrm{OMIM}$ ) both rely heavily on community input, but are both limited to special-interest groups.

The UniProt Knowledge Base (www. uniprot.org) provides an integrated view of gene functions. But the information is collected from highly heterogeneous experimental and computational data sources, so the annotations often lack confidence measures.

In addition, the entries in these genefunction databases - unlike those in Wikipedia - cannot be conveniently commented on or edited by average biologists. This discourages input from many potential contributors. Concise and accurate gene-function annotations compiled and edited by human experts are preferable in many cases.

A wiki on gene function, which utilizes the collective brain power of biologists around the world, would be an invaluable tool for biological sciences.

\section{Kai Wang}

Department of Microbiology,

University of Washington, Box 358070 ,

Seattle, Washington 98195, USA

\section{Conferences that welcome spouses aid research too}

SIR - In the struggle to balance career and family, long scientific conferences can represent a particular challenge. Occasionally, researchers may be tempted to invite their spouses. Not only is this a chance to spend time together, but it also provides an opportunity for the spouse to meet the key people in the researcher's field and put names together with faces.

This plan can present difficulties, as I recently discovered when I travelled with my wife and baby daughter to a Keystone conference my wife was attending in Utah. Although she encouraged us to join her for the social hour and dinner, we were told this daily event was "for scientists only". However, we expressed concern about this policy, and by the end of the week, the Keystone website announced that spouses would now be permitted to attend the evening social hours: a pleasing response.

Given the all-consuming nature of scientific research, adopting a more inclusive policy towards interested spouses would be a small gesture by every conference organizer that could go a surprisingly long way towards improving the happiness of researchers and of those who support them.

David A. Shaywitz

Department of Molecular and Cellular Biology, Harvard, and Neuroendocrine Unit, Bulfinch 457, Massachusetts General Hospital, 55 Fruit Street, Boston, Massachusetts 02114, USA

\section{Mudskippers undermine ID claims on macroevolution}

SIR - In his Science in Culture artide “Dying for a drink" (Nature 438, 564; 2005), Martin Kemp outlines the ancestral line of vertebrates, from modern humans back through evolutionary history to mudskippers.

Mudskippers are amphibious gobioids (a suborder of perch-related bony fishes) with arm-like pectoral fins. They live in the mangrove swamps of Africa, Asia and Australia, where they feed both in water and on land. The most terrestrial species catch insects and sometimes climb trees. They are living model organisms for the study of a key event in the history of life: the evolutionary transition of fishes to amphibians (tetrapods) that occurred about 364 million years ago.

Modern young-Earth creationists and adherents of the intelligent design (ID) movement have no problem with microevolution (speciation). But most of these Bible-based anti-darwinists refuse to accept macroevolution (phylogenetic development above the species level) on the grounds both that it is unscriptural and that it has never been observed (S.B. Carroll Nature 409, 669; 2001).

Mudskipping gobies and other amphibious fishes are examples of macroevolution in progress that can be analysed by observation and experiment. They are living intermediate forms that display a number of anatomical and physiological macromodifications of their fishlike body plan that enable them to live and forage on land.

These facts are relevant to the current ID debate, as they illustrate Darwin's dassical concept of descent with modification that evolved over past decades into the modern (synthetic) theory of biological evolution the unifying principle of all life sciences.

\section{U. Kutschera}

Institute of Biology, University of Kassel, Heinrich-Plett-Strasse 40,

D-34109 Kassel, Germany

\section{Reader-appeal should not outweigh merit of research}

SIR - There is one aspect of Nature's acceptance criteria that your Editorial on peer review ("Three cheers for peers" Nature 439,$118 ; 2006$ ) does not consider.

The broad audience of Nature forces its editors to pre-screen papers according to how appealing they will be for its readers, even if appeal and importance do not always go hand in hand. This is absolutely legitimate, given the broad character of the journal, given its independence (it is a private enterprise, after all) and given the fact that any author can choose whether to submit papers to Nature or not.

But special consideration is due to the growing weight that authoring papers in Nature is acquiring in personal curricula. Gratifying though this must be for Nature's editors, it has the slightly worrying implication that bright young scientists are beginning to be driven more by the appeal of a potential paper than by its importance a trend to which the scientific community should find a response.

The long and exemplary relationship between Nature and the scientific community allows me to hope that the journal itself will help in this endeavour.

Emilio Artacho

Department of EarthSciences,

University of Cambridge, Downing Street, Cambridge CB2 3EQ, UK 